Pacific

Journal of

Mathematics

\title{
CHORDAL GENERATORS AND \\ THE HYDRODYNAMIC NORMALIZATION \\ FOR THE UNIT BALL
}

SEbASTIAN SCHLEISSINGER 


\title{
CHORDAL GENERATORS AND THE HYDRODYNAMIC NORMALIZATION FOR THE UNIT BALL
}

\author{
SEbastian SchleisSinger
}

Let $c \geq 0$ and denote by $\mathcal{K}(\mathbb{H}, c)$ the set of all infinitesimal generators $G: \mathbb{C} \rightarrow \mathbb{C}$ on the upper half-plane $\mathbb{U}$ such that $\lim _{\sup } \sup _{y \rightarrow \infty} y \cdot|G(i y)| \leq c$. This class is related to univalent functions $f: \mathbb{H} \rightarrow \mathbb{W}$ with hydrodynamic normalization and appears in the so-called chordal Loewner equation.

In this paper, we generalize the class $\mathcal{K}(\mathbb{H}, c)$ and the hydrodynamic normalization to the Euclidean unit ball in $\mathbb{C}^{n}$. The generalization is based on the observation that $G \in \mathcal{K}(\mathbb{H}, c)$ can be characterized by an inequality for the hyperbolic length of $G(z)$.

1. Introduction 203

2. Chordal generators in higher dimensions 207

3. Slices 209

4. Univalent functions with hydrodynamic normalization 213

Appendix: Proof of Lemma 3.2 222

$\begin{array}{ll}\text { References } & 224\end{array}$

\section{Introduction}

One-parameter semigroups. Let $\mathbb{B}_{n}=\left\{z \in \mathbb{C}^{n} \mid\|z\|<1\right\}$ be the Euclidean unit ball in $\mathbb{C}^{n}$. In one dimension we write $\mathbb{D}:=\mathbb{B}_{1}$ for the unit disc.

Definition 1.1. A continuous one-real-parameter semigroup of holomorphic functions on $\mathbb{B}_{n}$ is a map $[0, \infty) \ni t \mapsto \Phi_{t} \in \mathcal{H}\left(\mathbb{B}_{n}, \mathbb{B}_{n}\right)$ satisfying the following conditions:

(1) $\Phi_{0}$ is the identity.

(2) $\Phi_{t+s}=\Phi_{t} \circ \Phi_{s}$ for all $t, s \geq 0$.

(3) $\Phi_{t}$ tends to the identity locally uniformly in $\mathbb{B}_{n}$, when $t$ tends to 0 .

Supported by the ERC grant "HEVO - Holomorphic Evolution Equations" n. 277691.

MSC2010: 20M20, 32A40, 37L05.

Keywords: semigroups of holomorphic mappings, infinitesimal generators, hydrodynamic normalization, chordal Loewner equation. 
Given such a semigroup $\left\{\Phi_{t}\right\}_{t \geq 0}$ and a point $z \in \mathbb{B}_{n}$, the limit

$$
G(z):=\lim _{t \rightarrow 0} \frac{\Phi_{t}(z)-z}{t}
$$

exists and the vector field $G: \mathbb{B}_{n} \rightarrow \mathbb{C}^{n}$, called the infinitesimal generator ${ }^{1}$ of $\Phi_{t}$, is a holomorphic function (see, e.g., [Abate 1992]). We denote by $\operatorname{Inf}\left(\mathbb{B}_{n}\right)$ the set of all infinitesimal generators of semigroups in $\mathbb{B}_{n}$. For any $z \in \mathbb{B}_{n}$, the map $w(t):=\Phi_{t}(z)$ is the solution of the initial value problem

$$
\frac{d w(t)}{d t}=G(w(t)), \quad w(0)=z .
$$

There are various characterizations of holomorphic functions $G: \mathbb{B}_{n} \rightarrow \mathbb{C}^{n}$ that are infinitesimal generators; see [Reich and Shoikhet 2005, Section 7.3], [Bracci et al. 2010, Theorem 0.2], [Bracci et al. 2014, p. 193].

The set $\operatorname{Inf}(\mathbb{D})$, i.e., all infinitesimal generators in the unit disc, can be characterized completely by the Berkson-Porta representation formula [1978]

$$
\begin{aligned}
\operatorname{Inf}(\mathbb{D})=\{z \mapsto(\tau-z)(1-\bar{\tau} z) p(z) \mid \tau \in \mathbb{\mathbb { D }}, p \in \mathcal{H}(\mathbb{D}, \mathbb{C}) & \\
& \text { with } \operatorname{Re}(p(z)) \geq 0 \text { for all } z \in \mathbb{D}\} .
\end{aligned}
$$

Remark 1.2. Let $F: \mathbb{D} \rightarrow \mathbb{D}$ be a holomorphic self-map. Recall the Denjoy-Wolff theorem (see, e.g., [Reich and Shoikhet 2005, Theorem 5.1]): If $F$ is not an elliptic automorphism (i.e., an automorphism with exactly one fixed point in $\mathbb{D}$ ), then there exists one point $\tau \in \overline{\mathbb{D}}$ (the Denjoy-Wolff point of $F$ ) such that the iterates $F^{n}$ converge locally uniformly in $\mathbb{D}$ to the constant map $\tau$.

If $\left\{\Phi_{t}\right\}_{t \geq 0}$ is a semigroup on $\mathbb{D}$, then we call $\tau \in \overline{\mathbb{D}}$ the Denjoy-Wolff point of $\left\{\Phi_{t}\right\}_{t \geq 0}$ if $\tau$ is the Denjoy-Wolff point of $\Phi_{1}$, which is equivalent to $\lim _{t \rightarrow \infty} \Phi_{t}=\tau$ locally uniformly.

If an infinitesimal generator in the unit disc does not generate a semigroup of elliptic automorphisms of $\mathbb{D}$, then the point $\tau \in \overline{\mathbb{D}}$ from formula (1-2) is exactly the Denjoy-Wolff point of the semigroup.

There are two special cases of infinitesimal generators in $\mathbb{D}$ that have been studied intensively and turned out to be quite useful in Loewner theory and its applications. The two different cases arise from certain normalizations of the Berkson-Porta data $\tau$ and $p$ from formula (1-2). In the radial case, one considers those elements $G \in \operatorname{Inf}(\mathbb{D})$ whose Berkson-Porta data $\tau$ and $p$ satisfy

$$
\tau=0 \quad \text { and } \quad p(0)=1 \text {, }
$$

i.e., $G(z)=-z p(z)$.

\footnotetext{
${ }^{1}$ There is no standard convention in the literature and often $-G$ is called the infinitesimal generator of the semigroup.
} 
This class plays a central role in studying the class $S$ of all univalent functions $f: \mathbb{D} \rightarrow \mathbb{C}$ with $f(0)=0, f^{\prime}(0)=1$, via the powerful tools of Loewner's theory, which considers a nonautonomous version of (1-1); see, e.g., [Pommerenke 1975, Chapter 6]. The class of radial generators as well as the class $S$ have been generalized in this context to the polydisc $\mathbb{D}^{n}$ (see [Poreda 1987a; 1987b]), and to the unit ball $\mathbb{B}_{n}$ (see [Graham and Kohr 2003] for a collection of several results and references).

The second class, the set of all chordal generators ${ }^{2}$, consists of all $G \in \operatorname{Inf}(\mathbb{D})$ whose Berkson-Porta data $\tau$ and $p$ satisfy

$$
\tau=1 \quad \text { and } \quad \angle \lim _{z \rightarrow 1} \frac{p(z)}{z-1} \text { is finite. }
$$

The aim of this paper is to introduce a generalization of the chordal class for the unit ball $\mathbb{B}_{n}$.

The hydrodynamic normalization in one dimension. Instead of fixing an interior point, like in the class $S$, it can be of interest to investigate univalent self-mappings of $\mathbb{D}$ that fix a boundary point. In this case, one usually passes from $\mathbb{D}$ to the upper half-plane $\mathbb{H}=\{z \in \mathbb{C} \mid \operatorname{Im}(z)>0\}$.

A class of such mappings that is easy to describe and that appears in several applications is the set of all univalent mappings $f: \mathbb{W} \rightarrow \mathbb{W}$ that fix the boundary point $\infty$ and have the so-called hydrodynamic normalization. Basic properties of this class can be found in [Goryaŭnov and Ba 1992]; see also [Bauer 2005; Contreras et al. 2010]. One of its main applications is the chordal Loewner equation; see [Abate et al. 2010, Section 4] for further references.

A univalent function $f: \mathbb{W} \rightarrow \mathbb{W}$ has hydrodynamic normalization (at $\infty$ ) if $f$ has the expansion

$$
f(z)=z-\frac{c}{z}+\gamma(z),
$$

where $c \geq 0$, which is usually called half-plane capacity, and $\gamma$ satisfies

$$
\angle \lim _{z \rightarrow \infty} z \cdot \gamma(z)=0 .
$$

We denote by $\mathfrak{P}$ the set of all these functions. Then $\mathfrak{P}$ is a semigroup and the functional $l: \mathfrak{P} \rightarrow[0, \infty), l(f)=c$, is additive: if $f_{1}, f_{2} \in \mathfrak{P}$, then $f_{1} \circ f_{2} \in \mathfrak{P}$ and $l\left(f_{1} \circ f_{2}\right)=l\left(f_{1}\right)+l\left(f_{2}\right)$.

Remark 1.3. Let $f \in \mathfrak{P}$ with $l(f)=c$. If we transfer $f$ to the unit disc by conjugation by the Cayley transform, then we obtain a function $\tilde{f}: \mathbb{D} \rightarrow \mathbb{D}$ having

2 Note that there is no standard use of the words "radial" and "chordal" in the literature. In [Contreras et al. 2010], e.g., an element $G \in \operatorname{Inf}(\mathbb{D})$ is called radial if $\tau \in \mathbb{D}$ and chordal if $\tau \in \partial \mathbb{D}$. 
the expansion

$$
\tilde{f}(z)=z-\frac{c}{4}(z-1)^{3}+\tilde{\gamma}(z),
$$

where $\angle \lim _{z \rightarrow 1} \tilde{\gamma}(z) /(z-1)^{3}=0$.

If $\left\{\Phi_{t}\right\}_{t \geq 0}$ is a one-real-parameter semigroup contained in $\mathfrak{P}$ with $l\left(\Phi_{1}\right)=a$, then it is easy to see that $l\left(\Phi_{t}\right)=a \cdot t$. If $H$ is the generator of this semigroup, then we also define $l(H):=a$.

We will be interested in the following set of chordal generators.

Definition 1.4. By $\mathcal{K}(\mathbb{H}, c)$ we denote the set of all infinitesimal generators $H$ of one-real-parameter semigroups $\left\{\Phi_{t}\right\}_{t \geq 0}$ contained in $\mathfrak{P}$ with $l(H) \leq c$.

Remark 1.5. The set $\mathcal{K}(\mathbb{H}, c)$ can be characterized in various ways; see [Goryaĭnov and Ba 1992, Section 1] and [Maassen 1992, Proposition 2.2].

It is known that $H \in \mathcal{K}(\mathbb{H}, c)$ for some $c \geq 0$ if and only if $H$ maps $\mathbb{W}$ into $\overline{\mathbb{M}}$ and

$$
\limsup _{y \rightarrow \infty} y|H(i y)| \leq c .
$$

In fact, $l(H)=\limsup _{y \rightarrow \infty} y|H(i y)|$.

Furthermore, this is equivalent to $H$ maps $\mathbb{H}$ into $\overline{\mathbb{E}}$ and

$$
|H(z)| \leq \frac{c}{\operatorname{Im}(z)}
$$

for all $z \in \mathbb{H}$. The number $l(H)$ is the smallest constant such that this inequality holds.

Finally, it is known that this property is equivalent to the fact that $-G$ is the Cauchy transform of a finite, nonnegative Borel measure $\mu$ on $\mathbb{R}$, i.e.,

$$
H(z)=\int_{\mathbb{R}} \frac{\mu(d u)}{u-z} .
$$

The number $l(H)$ can be calculated by $l(H)=\mu(\mathbb{R})$.

Remark 1.6. It is easy to see that the following holds: if $f \in \mathfrak{P}$ with $c=l(f)$, then $H:=f-\mathrm{id} \in \mathcal{K}(\mathbb{H}, c)$ with $l(H)=c$.

Let $C: \mathbb{H} \rightarrow \mathbb{D}, C(z)=(z-i) /(z+i)$, be the Cayley map. We define $\mathcal{K}(\mathbb{D}, c)$ by

$$
\mathcal{K}(\mathbb{D}, c)=\left\{C^{\prime}\left(C^{-1}\right) \cdot\left(H \circ C^{-1}\right) \mid H \in \mathcal{K}(\mathbb{H}, c)\right\}^{3}
$$

The rest of this paper is organized as follows: In Section 2 we look for an invariant characterization of chordal generators, i.e., of the sets $\mathcal{K}(\mathbb{H}, c)$ and $\mathcal{K}(\mathbb{D}, c)$, and we introduce the class $\mathcal{K}\left(\mathbb{B}_{n}, c\right)$ for the higher-dimensional unit ball. It will turn out to be quite useful to study "slices" of this class, which is done in Section 3. In Section 4 we introduce and study the class $\mathfrak{P}_{n}$, a higher-dimensional analog of the class $\mathfrak{P}$.

\footnotetext{
${ }^{3}$ If $\left\{\Phi_{t}\right\}_{t \geq 0}$ is a semigroup in $\mathbb{U}$ with generator $H$, then $\left\{C \circ \Phi_{t} \circ C^{-1}\right\}_{t \geq 0}$ is a semigroup in $\mathbb{D}$ and its generator is given by $C^{\prime}\left(C^{-1}\right) \cdot\left(H \circ C^{-1}\right)$.
} 


\section{Chordal generators in higher dimensions}

Invariant formulation for $\mathcal{K}(\mathbb{D}, \boldsymbol{c})$ and $\mathcal{K}(\mathbb{W}, \boldsymbol{c})$. For $R>0$, we let $E_{\mathbb{D}}(1, R)$ be the horodisc in $\mathbb{D}$ with center 1 and radius $R$, i.e.,

$$
E_{\mathbb{D}}(1, R)=\left\{z \in \mathbb{D} \mid \frac{1}{\left|u_{\mathbb{D}}(z)\right|}<R\right\},
$$

where $u_{\mathbb{D}}(z)=-\left(1-|z|^{2}\right) /|1-z|^{2}$ is the Poisson kernel in $\mathbb{D}$ with respect to 1 .

By using the Cayley map, we define analogously

$$
E_{\mathbb{\sharp}}(\infty, R)=C^{-1}\left(E_{\mathbb{D}}(1, R)\right)=\left\{z \in \mathbb{U} \mid \frac{1}{\operatorname{Im}(z)}<R\right\} .
$$

For $z \in \mathbb{D}$ and a tangent vector $v \in \mathbb{C}$, we denote by $|v|_{\mathbb{D}, z}$ the hyperbolic length of $v$, i.e.,

$$
|v|_{\mathbb{D}, z}:=\frac{2|v|}{1-|z|^{2}} .
$$

Furthermore, we let $R_{\mathbb{D}}(z)$ be the radius $R$ of the horodisc $E_{\mathbb{D}}(1, R)$ that satisfies $z \in \partial E(1, R)$; in short, $R_{\mathbb{D}}(z)=1 /\left|u_{\mathbb{D}}(z)\right|$. Analogously, for $z \in \mathbb{U}$ and $v \in \mathbb{C}$, we

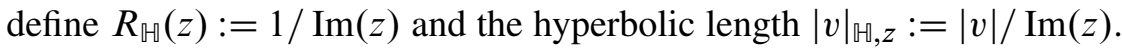

According to (1-4), we know that $H \in \mathcal{K}(\mathbb{W}, c)$ if and only if $H$ maps $\mathbb{W}$ into $\overline{\mathbb{W}}$ and $|H(z)| \leq c / \operatorname{Im}(z)$ for all $z \in \mathbb{H}$. By using the Berkson-Porta formula, it is easy to see that we can rephrase this to: $H \in \mathcal{K}(\mathbb{H}, c)$ if and only if $H \in \operatorname{Inf}(\mathbb{H})$ and $|H(z)| \leq c / \operatorname{Im}(z)$ for all $z \in \mathbb{H}$.

The last inequality is equivalent to $|H(z)| / \operatorname{Im}(z) \leq c / \operatorname{Im}(z)^{2}$ or

$$
|H(z)|_{\mathbb{\sharp}, z} \leq \frac{c}{\operatorname{Im}(z)^{2}}=c \cdot R_{\mathbb{\sharp}}(z)^{2} .
$$

If we pass from $\mathbb{H}$ to $\mathbb{D}$ and transform $H$ into $G=C^{\prime}\left(C^{-1}\right) \cdot\left(H \circ C^{-1}\right)$,

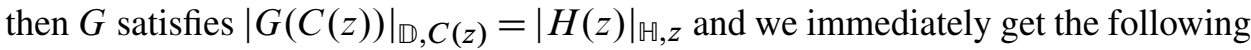
characterization.

Proposition 2.1. Let $G \in \operatorname{Inf}(\mathbb{D})$. Then

$$
G \in \mathcal{K}(\mathbb{D}, c) \Longleftrightarrow|G(z)|_{\mathbb{D}, z} \leq c \cdot R_{\mathbb{D}}(z)^{2} \text { for all } z \in \mathbb{D} .
$$

Let $H \in \operatorname{Inf}(\mathbb{M})$. Then

$$
H \in \mathcal{K}(\mathbb{H}, c) \Longleftrightarrow|H(z)|_{\mathbb{H}, z} \leq c \cdot R_{\mathbb{H}}(z)^{2} \text { for all } z \in \mathbb{H} .
$$

Chordal generators in the unit ball. For $n \in \mathbb{N}$, let $u_{n}$ be the pluricomplex Poisson kernel in $\mathbb{B}_{n}$ with pole at $e_{1}:=(1,0, \ldots, 0)$, i.e.,

$$
u_{\mathbb{B}_{n}, p}=-\frac{1-\|z\|^{2}}{\left|1-z_{1}\right|^{2}} .
$$


The level sets of $u_{\mathbb{B}_{n}}$ are exactly the boundaries of horospheres with center $e_{1}$; more precisely, the set

$$
E_{\mathbb{B}_{n}}\left(e_{1}, R\right):=\left\{\left.z \in \mathbb{B}_{n}|| u_{\mathbb{B}_{n}}(z)\right|^{-1}<R\right\}, \quad R>0,
$$

is the horosphere with center $e_{1}$ and radius $R$.

Furthermore, for $z \in \mathbb{B}_{n}$ and $v \in \mathbb{C}^{n}$, we denote by $\|v\|_{\mathbb{B}_{n}, z}$ the Kobayashihyperbolic length of the vector $v$ with respect to $z$.

Motivated by Proposition 2.1, we make the following definition.

Definition 2.2. Let $c \geq 0$. We define the class $\mathcal{K}\left(\mathbb{B}_{n}, c\right)$ to be the set of all infinitesimal generators $G$ on $\mathbb{B}_{n}$ such that, for all $z \in \mathbb{B}_{n}$,

$$
\|G(z)\|_{\mathbb{B}_{n}, z} \leq \frac{c}{u_{\mathbb{B}_{n}}(z)^{2}} .
$$

Remark 2.3. $\mathcal{K}\left(\mathbb{B}_{n}, c\right)$ is a compact family: Montel's theorem and the definition of $\mathcal{K}\left(\mathbb{B}_{n}, c\right)$ immediately imply that it is a normal family. If a sequence $\left(G_{n}\right) \subset$ $\mathcal{K}\left(\mathbb{B}_{n}, c\right)$ converges locally uniformly to $G: \mathbb{B}_{n} \rightarrow \mathbb{C}^{n}$, then $G$ is holomorphic and also an infinitesimal generator, which can be seen by using the characterization given in [Bracci et al. 2010, Theorem 0.2]. Of course, $G$ also satisfies (2-1) and we conclude $G \in \mathcal{K}\left(\mathbb{B}_{n}, c\right)$.

Just as we passed from $\mathbb{D}$ to $\mathbb{E}$ in one dimension, we can pass from the unit ball $\mathbb{B}_{n}$ to the Siegel upper half-space $\mathbb{H}_{n}=\left\{\left(z_{1}, \tilde{z}\right) \in \mathbb{C}^{n} \mid \operatorname{Im}\left(z_{1}\right)>\|\tilde{z}\|^{2}\right\}$ in order to get simpler formulas:

The Cayley map

$$
C: \mathbb{H}_{n} \rightarrow \mathbb{B}_{n}, \quad C(z)=\left(C_{1}(z), \ldots, C_{n}(z)\right)=\left(\frac{z_{1}-i}{z_{1}+i}, \frac{2 z_{2}}{z_{1}+i}, \ldots, \frac{2 z_{n}}{z_{1}+i}\right),
$$

maps $\mathbb{H}_{n}$ biholomorphically onto $\mathbb{B}_{n}$. It extends to a homeomorphism from the one-point compactification $\widehat{\mathbb{H}}_{n}=\mathbb{U}_{n} \cup \partial \mathbb{H}_{n} \cup\{\infty\}$ of $\mathbb{U}_{n} \cup \partial \mathbb{W}_{n}$ to the closure of $\mathbb{B}^{n}$.

The pluricomplex Poisson kernel transforms as follows:

$$
u_{\sharp_{n}}(z):=u_{\mathbb{B}_{n}}(C(z))=-\operatorname{Im}\left(z_{1}\right)+\|\tilde{z}\|^{2} .
$$

Thus, we define the horosphere $E_{\uplus_{n}}(\infty, R)$ with center $\infty$ and radius $R>0$ by

$$
E_{\mathbb{H}_{n}}(\infty, R):=\left\{z \in \mathbb{H}_{n} \mid \operatorname{Im}\left(z_{1}\right)-\|\tilde{z}\|^{2}>\frac{1}{R}\right\} .
$$

For $v \in \mathbb{C}^{n}$ and $z \in \mathbb{H}_{n}$, we let $\|v\|_{\mathbb{H}_{n}, z}$ be the Kobayashi hyperbolic length of $v$.

Let $c \geq 0$. We define the class $\mathcal{K}\left(\mathbb{H}_{n}, c\right)$ to be the set of all infinitesimal generators $H$ on $\mathbb{H}_{n}$ satisfying the inequality

$$
\|H(z)\|_{\mathbb{H}_{n}, z} \leq \frac{c}{u_{\mathbb{\boxplus}_{n}}(z)^{2}}
$$


for all $z \in \mathbb{H}_{n}$. Then we have

$$
\mathcal{K}\left(\mathbb{B}_{n}, c\right)=\left\{C^{\prime}\left(C^{-1}\right) \cdot\left(H \circ C^{-1}\right) \mid H \in \mathcal{K}\left(\mathbb{H}_{n}, c\right)\right\} .
$$

From now on we will stay in the upper half-space $\mathbb{H}_{n}$, where most of the computations we need take a simpler form.

\section{Slices}

Normalized geodesics and slices. For any $H \in \operatorname{Inf}\left(\mathbb{H}_{n}\right)$, one can consider onedimensional slices by using the so-called Lempert projection devices; see [Bracci and Shoikhet 2014, Section 3].

If $w \in \mathbb{H}_{n}$, then there exists a unique complex geodesic passing through $w$ and $\infty$. Let us choose a parametrization $\varphi: \mathbb{H} \rightarrow \mathbb{H}_{n}$ of this geodesic. There exists a unique holomorphic map $P: \mathbb{H}_{n} \rightarrow \mathbb{H}_{n}$ with $P^{2}=P$ and $P \circ \varphi=\varphi$. Define $\widetilde{P}=\varphi^{-1} \circ P$. Then

$$
h_{\varphi}: \mathbb{H} \rightarrow \mathbb{C}, \quad h_{\varphi}(\zeta)=d \widetilde{P}(\varphi(\zeta)) \cdot H(\varphi(\zeta)),
$$

is an infinitesimal generator on $\mathbb{H}$; see [Bracci and Shoikhet 2014, p. 6].

We will need special parametrizations of these geodesics: In [Bracci and Patrizio 2005, p. 516], it is shown that for any complex geodesic $\varphi: \mathbb{H} \rightarrow \mathbb{H}_{n}$ with $\varphi(\infty)=\infty$, there exists $a_{\varphi}>0$ such that

$$
u_{\mathbb{\sharp}}(\varphi(\zeta))=a_{\varphi} \cdot u_{\llbracket}(\zeta)
$$

for all $\zeta \in \mathbb{M}$. Call a geodesic $\varphi: \mathbb{H} \rightarrow \mathbb{H}_{n}$ normalized if $\varphi(\infty)=\infty$ and $a_{\varphi}=1$.

Lemma 3.1. Let $a \in \mathbb{C}$ and $\gamma \in \mathbb{C}^{n-1}$ such that $(a, \gamma) \in \mathbb{H}_{n}$. Then the map

$$
\varphi_{\gamma}: \mathbb{H} \rightarrow \mathbb{H}_{n}, \quad \varphi_{\gamma}(\zeta):=\left(\zeta+i\|\gamma\|^{2}, \gamma\right),
$$

is a normalized geodesic through $(a, \gamma)$. Furthermore, if $H=\left(H_{1}, \widetilde{H}\right) \in \operatorname{Inf}\left(\mathbb{H}_{n}\right)$, then the slice $h_{\gamma}:=h_{\varphi_{\gamma}}$ of $H$ with respect to $\varphi_{\gamma}$ is given by

$$
h_{\gamma}(\zeta)=H_{1}\left(\varphi_{\gamma}(\zeta)\right)-2 i \bar{\gamma}^{T} \cdot \tilde{H}\left(\varphi_{\gamma}(\zeta)\right)
$$

Proof. Let $\psi: \mathbb{D} \rightarrow \mathbb{B}_{n}$ be a complex geodesic with $\psi(1)=e_{1}$. As a parametrization for $\psi$, one can choose (see [Bracci and Shoikhet 2014, Section 3])

$$
\psi(\zeta)=\left(\alpha^{2}(\zeta-1)+1, \alpha(\zeta-1) \beta\right),
$$

where $\alpha>0$ and $\beta \in \mathbb{C}^{n-1}$ such that $\|\beta\|^{2}=1-\alpha^{2}$. Then

$$
C^{-1}(\psi(\zeta))=\left(i \frac{2+\alpha^{2}(\zeta-1)}{\alpha^{2}(1-\zeta)}, i \beta / \alpha\right)
$$


and

$$
\begin{aligned}
\zeta \mapsto C^{-1}\left(\psi\left(C_{1}(\zeta)\right)\right) & =\left(-i+\frac{\zeta+i}{\alpha^{2}}, i \beta / \alpha\right) \\
& =\left(\frac{\zeta}{\alpha^{2}}+i \frac{1-\alpha^{2}}{\alpha^{2}}, i \beta / \alpha\right)=\left(\frac{\zeta}{\alpha^{2}}+i\left\|\frac{\beta}{\alpha}\right\|^{2}, i \beta / \alpha\right)
\end{aligned}
$$

is a complex geodesic from $\mathbb{U}$ to $\mathbb{U}_{n}$. A reparametrization $\left(\zeta / \alpha^{2}\right.$ to $\left.\zeta\right)$ and setting $\gamma=i \beta / \alpha$ gives the geodesic

$$
\varphi_{\gamma}(\zeta)=\left(\zeta+i\|\gamma\|^{2}, \gamma\right)
$$

This complex geodesic is normalized because it satisfies $\varphi_{\gamma}(\infty)=\infty$ and

$$
u_{\sharp \sharp_{n}}\left(\varphi_{\gamma}(\zeta)\right)=\operatorname{Im}\left(\zeta+i\|\gamma\|^{2}\right)-\|\gamma\|^{2}=\operatorname{Im}(\zeta)=u_{\sharp}(\zeta) .
$$

The projection onto $\varphi_{\gamma}(\mathbb{M})$ is given by

$$
P\left(z_{1}, \tilde{z}\right)=\left(z_{1}-2 i \bar{\gamma}^{T} \cdot \tilde{z}+2 i\|\gamma\|^{2}, \gamma\right) .
$$

Clearly, $P$ is holomorphic and maps $\mathbb{H}_{n}$ onto $\varphi_{\gamma}(\mathbb{W})$ because

$$
\begin{aligned}
\operatorname{Im}\left(z_{1}-2 i \bar{\gamma}^{T} \cdot \tilde{z}+2 i\|\gamma\|^{2}\right) & =\operatorname{Im}\left(z_{1}\right)-2 \operatorname{Im}\left(i \bar{\gamma}^{T} \cdot \tilde{z}\right)+2\|\gamma\|^{2} \\
& \geq\|\tilde{z}\|^{2}-2\|\gamma\|\|\tilde{z}\|+\|\gamma\|^{2}+\|\gamma\|^{2} \\
& =(\|\gamma\|-\|\tilde{z}\|)^{2}+\|\gamma\|^{2} \geq\|\gamma\|^{2} .
\end{aligned}
$$

Furthermore,

$$
\begin{aligned}
(P \circ P)\left(z_{1}, \tilde{z}\right) & =\left(z_{1}-2 i \bar{\gamma}^{T} \tilde{z}+2 i\|\gamma\|^{2}-2 i \bar{\gamma}^{T} \gamma+2 i\|\gamma\|^{2}, \gamma\right) \\
& =\left(z_{1}-2 i \bar{\gamma}^{T} \tilde{z}+2 i\|\gamma\|^{2}, \gamma\right)=P\left(z_{1}, \tilde{z}\right) .
\end{aligned}
$$

Thus, the inverse $\widetilde{P}: \mathbb{H}_{2} \rightarrow \mathbb{H}, \widetilde{P}=\varphi_{\gamma}^{-1} \circ P$, is given by

$$
\widetilde{P}\left(z_{1}, \tilde{z}\right)=\left(z_{1}-2 i \bar{\gamma}^{T} \tilde{z}+i\|\gamma\|^{2}\right) .
$$

If $H(z)=\left(H_{1}(z), \widetilde{H}(z)\right)$ is a generator on $\mathbb{H}_{n}$, we get the slice reduction

$$
\begin{aligned}
h_{\varphi_{\gamma}}(\zeta) & =d \widetilde{P}\left(\varphi_{\gamma}(\zeta)\right) \cdot H\left(\varphi_{\gamma}(\zeta)\right) \\
& =H_{1}\left(\varphi_{\gamma}(\zeta)\right)-2 i \bar{\gamma}^{T} \cdot \widetilde{H}\left(\varphi_{\gamma}(\zeta)\right)
\end{aligned}
$$

Some explicit formulas. Later on we will need explicit formulas of the Kobayashi norms of $d P(z) H(z)$ and $H(z)-d P(z) \cdot H(z)$. The following lemma is proven in the Appendix. 
Lemma 3.2. Let $a \in \mathbb{C}, p, v \in \mathbb{C}^{n-1}$ and $z=\left(z_{1}, \tilde{z}\right) \in \mathbb{M}_{n}$. Then the following formulas hold:

$$
\left\|\left(\begin{array}{c}
a \\
0
\end{array}\right)\right\|_{\mathbb{H}_{n}, z}=\frac{|a|}{\left|u_{\mathbb{H}_{n}}(z)\right|}
$$

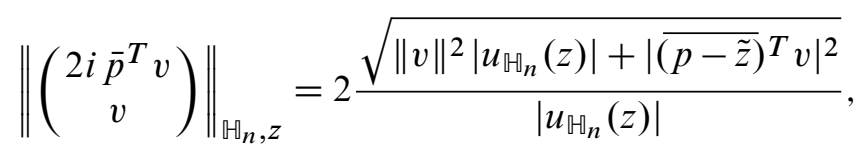

$$
\left\|\left(\begin{array}{c}
a-2 i \overline{\tilde{z}}^{T} v \\
0
\end{array}\right)+\left(\begin{array}{c}
2 i \overline{\tilde{z}}^{T} v \\
v
\end{array}\right)\right\|_{\mathbb{H}_{n}, z}^{2}=\left\|\left(\begin{array}{c}
a-2 i \overline{\tilde{z}}^{T} v \\
0
\end{array}\right)\right\|_{\mathbb{H}_{n}, z}^{2}+\left\|\left(\begin{array}{c}
2 i \overline{\tilde{z}}^{T} v \\
v
\end{array}\right)\right\|_{\mathbb{H}_{n}, z}^{2} .
$$

By using Lemma 3.2 we obtain the following explicit expressions.

Lemma 3.3. Let $H=\left(H_{1}, \tilde{H}\right) \in \operatorname{Inf}\left(\mathbb{H}_{n}\right)$ and fix $z \in \mathbb{H}_{n}$. Denote by $P$ the projection onto the complex geodesic through $z$ and $\infty$. Then the following formulas hold:

$$
\begin{gathered}
d P(z) \cdot H(z)=\left(H_{1}(z)-2 i \overline{\tilde{z}}^{T} \tilde{H}(z), 0\right), \\
H(z)-d P(z) \cdot H(z)=\left(2 i \overline{\tilde{z}}^{T} \tilde{H}(z), \tilde{H}(z)\right) .
\end{gathered}
$$

Furthermore,

$$
\begin{aligned}
& \|H(z)\|_{\uplus_{n}, z}^{2}=\|d P(z) \cdot H(z)\|_{\boxplus_{n}, z}^{2}+\|H(z)-d P(z) \cdot H(z)\|_{\boxplus_{n}, z}^{2}, \\
& \|d P(z) H(z)\|_{\boxplus_{n}, z}=\frac{\left|H_{1}(z)-2 i \overline{\tilde{z}}^{T} \tilde{H}(z)\right|}{\left|u_{\boxplus_{n}}(z)\right|}, \\
& \|H(z)-d P(z) \cdot H(z)\|_{\boxplus_{n}, z}=2 \frac{\|\tilde{H}(z)\|}{\sqrt{\left|u_{\boxplus_{n}}(z)\right|}} .
\end{aligned}
$$

Proof. The formulas for $d P(z) H(z)$ and $H(z)-d P(z) H(z)$ follow from the explicit form (3-3).

Equation (3-8) follows from (3-6) with $a=H_{1}(z)$ and $v=\widetilde{H}(z)$.

Furthermore, (3-9) follows directly from (3-4) with $a=H_{1}(z)-2 i \overline{\tilde{z}}^{T} \tilde{H}(z)$ and (3-10) from (3-5) by setting $p=\tilde{z}$ and $v=\tilde{H}$.

Slices of generators in $\mathcal{K}\left(\mathbb{\boxplus}_{n}, c\right)$ and examples.

Proposition 3.4. Let $c \geq 0$ and $H \in \mathcal{K}\left(\mathbb{H}_{n}, c\right)$. Then every normalized slice $h_{\gamma}$ of $H$ belongs to $\mathcal{K}(\mathbb{M}, c)$.

Proof. Fix $\gamma \in \mathbb{C}^{n-1}$ and $\zeta \in \mathbb{H}$ and let $z=\varphi_{\gamma}(\zeta)$.

Furthermore, let $P$ be the projection onto $\varphi_{\gamma}(\mathbb{M})$. Now we write $H(z)$ as

$$
H(z)=d P(z) \cdot H(z)+(H(z)-d P(z) H(z)) .
$$


As $H \in \mathcal{K}\left(\mathbb{H}_{n}, c\right)$, equation (3-8) implies

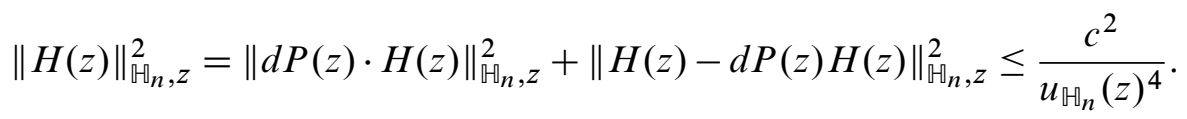

In particular,

$$
\|d P(z) \cdot H(z)\|_{\mathbb{\boxplus}_{n}, z} \leq \frac{c}{u_{\mathbb{\boxplus}_{n}}(z)^{2}} .
$$

By the definition of the slice $h_{\gamma}$, we have

$$
d P\left(\varphi_{\gamma}(\zeta)\right) \cdot H\left(\varphi_{\gamma}(\zeta)\right)=\left(d \varphi_{\gamma}\right)(\zeta) \cdot h_{\gamma}(\zeta)
$$

and consequently

$$
\left\|d P\left(\varphi_{\gamma}(\zeta)\right) \cdot H\left(\varphi_{\gamma}(z)\right)\right\|_{\mathbb{\boxplus}_{n}, \varphi_{\gamma}(\zeta)}=\left\|\left(d \varphi_{\gamma}\right)(\zeta) \cdot h_{\gamma}(\zeta)\right\|_{\mathbb{\boxplus}_{n}, \varphi_{\gamma}(\zeta)}=\left|h_{\gamma}(\zeta)\right|_{\mathbb{H}, \zeta} \cdot
$$

The last equality holds as $\varphi_{\gamma}$ is a complex geodesic. Equation (3-11) implies

$$
\left|h_{\gamma}(\zeta)\right|_{\mathbb{\Vdash}, \zeta} \leq \frac{c}{u_{\mathbb{\sharp}}\left(\varphi_{\gamma}(\zeta)\right)^{2}}=\frac{c}{u_{\mathbb{}}(\zeta)^{2}},
$$

where the last equality holds as $\varphi_{\gamma}$ is normalized. Hence, $h_{\gamma} \in \mathcal{K}(\mathbb{H}, c)$.

Remark 3.5. If two holomorphic functions $H_{1}, H_{2}: \mathbb{W}_{n} \rightarrow \mathbb{C}^{n}$ have the same slices, i.e., $d P(z) H_{1}(z)=d P(z) H_{2}(z)$ for all $z \in \mathbb{U}_{n}$, then $H_{1}=H_{2}$; see the proof of Theorem 3.2 in [Casavecchia 2010].

Example 3.6. The family $\left\{\Phi_{t}(z)=\left(z_{1}, e^{-i t / z_{1}} z_{2}\right)\right\}_{t \geq 0}$ is a semigroup on $\mathbb{H}_{2}$. Its generator $H$ is given by

$$
H\left(z_{1}, z_{2}\right)=\left(0,-i \frac{z_{2}}{z_{1}}\right) .
$$

Thus, for $\gamma \in \mathbb{C}$, the slice $h_{\gamma}$ has the form

$$
h_{\gamma}(z)=-2 i \bar{\gamma} \cdot-i \frac{\gamma}{z+i|\gamma|^{2}}=\frac{-2|\gamma|^{2}}{z+i|\gamma|^{2}} .
$$

Consequently, the $\operatorname{limit}_{y \rightarrow \infty} y \cdot|h(i y)|=2|\gamma|^{2}$ exists, but does not have an upper bound that is independent of $\gamma$. Proposition 3.4 implies that for any $c \geq 0$, $H \notin \mathcal{K}\left(\mathbb{M}_{2}, c\right)$.

Example 3.7. Let

$$
H: \mathbb{H}_{2} \rightarrow \mathbb{C}^{2}, \quad H\left(z_{1}, z_{2}\right)=\left(\begin{array}{c}
-1 / z_{1} \\
z_{2} / 2 z_{1}^{2}
\end{array}\right) .
$$


For $\gamma \in \mathbb{C}$, the slice $h_{\gamma}$ is given by

$$
\begin{aligned}
h_{\gamma}(\zeta) & =\frac{-1}{\zeta+i|\gamma|^{2}}-2 i \bar{\gamma} \cdot \frac{\gamma}{2\left(\zeta+i|\gamma|^{2}\right)^{2}} \\
& =\frac{-\zeta-2 i|\gamma|^{2}}{\left(\zeta+i|\gamma|^{2}\right)^{2}}=\frac{\left(-\zeta-2 i|\gamma|^{2}\right)\left(\bar{\zeta}^{2}-2 i|\gamma|^{2} \bar{\zeta}-|\gamma|^{4}\right)}{\left.\left.|\zeta+i| \gamma\right|^{2}\right|^{4}} .
\end{aligned}
$$

Let us write $\zeta=x+i y, x \in \mathbb{R}, y \in(0, \infty)$. Then a small calculation gives

$$
\operatorname{Im}\left(h_{\gamma}(\zeta)\right)=\frac{y\left(x^{2}+y^{2}\right)+4 y^{2}|\gamma|^{2}+5 y|\gamma|^{4}+2|\gamma|^{6}}{\left.\left.|\zeta+i| \gamma\right|^{2}\right|^{4}}>0 .
$$

Furthermore,

$$
\limsup _{y \rightarrow \infty} y\left|h_{\gamma}(i y)\right|=1 .
$$

Hence, $h_{\gamma} \in \mathcal{K}(\mathbb{M}, 1)$. So each slice is an infinitesimal generator in $\mathbb{W}$ and by [Bracci and Shoikhet 2014, Proposition 3.8], the function $H$ is an infinitesimal generator in $\mathbb{H}_{2}$.

Now let $\left(z_{1}, z_{2}\right) \in \mathbb{H}_{2}$ and write $z_{1}=x+i y, x, y \in \mathbb{R}$. Then we get

$$
\begin{aligned}
u_{\mathbb{\boxplus}_{2}}(z)^{4} \cdot\|H(z)\|_{\mathbb{\boxplus}_{2}, z}^{2} & =\left(y-\left|z_{2}\right|^{2}\right)^{2} \cdot \frac{x^{2}+y^{2}+3\left|z_{2}\right|^{2} y}{\left(x^{2}+y^{2}\right)^{2}} \\
& \leq y_{y \geq\left|z_{2}\right|^{2}} y^{2} \cdot \frac{x^{2}+y^{2}+3 y^{2}}{\left(x^{2}+y^{2}\right)^{2}} \leq \frac{x^{2}+4 y^{2}}{x^{2}+y^{2}} \leq 4
\end{aligned}
$$

(an explicit formula of the Kobayashi metric is given in the Appendix). Consequently, $H \in \mathcal{K}\left(\mathbb{H}_{2}, 2\right)$.

Question 3.8. Let $H: \mathbb{U}_{n} \rightarrow \mathbb{C}^{n}$ be an infinitesimal generator. Assume there exists $c \geq 0$ such that $h_{\gamma} \in \mathcal{K}(\mathbb{M}, c)$ for every $\gamma \in \mathbb{C}^{n-1}$. Does this imply that $H \in \mathcal{K}\left(\mathbb{H}_{n}, C\right)$ for some $C \geq c$ ?

\section{Univalent functions with hydrodynamic normalization}

Motivated by Remark 1.6, we define the following generalization of the class $\mathfrak{P}$, where id stands for the identity mapping on $\mathbb{H}_{n}$.

\section{Definition 4.1.}

$\mathfrak{P}_{n}:=\left\{f: \mathbb{M}_{n} \rightarrow \mathbb{M}_{n} \mid f\right.$ is univalent and $f-\mathrm{id} \in \mathcal{K}\left(\mathbb{M}_{n}, c\right)$ for some $\left.c \geq 0\right\}$.

Remark 4.2. It is important to note that if $f: \mathbb{H}_{n} \rightarrow \mathbb{H}_{n}$ is a holomorphic selfmapping, then the map $f$-id is automatically an infinitesimal generator; see [Reich and Shoikhet 2005, p. 207]. 
Basic properties of $\mathfrak{P}_{\boldsymbol{n}}$. The following proposition summarizes some basic properties of $\mathfrak{P}_{n}$.

Proposition 4.3. (a) $\mathfrak{P}_{n}$ contains no automorphism of $\mathbb{U}_{n}$ except the identity.

(b) Let $\alpha: \mathbb{M}_{n} \rightarrow \mathbb{U}_{n}$ be an automorphism of $\mathbb{U}_{n}$ with $\alpha(\infty)=\infty$. If $f \in \mathfrak{P}_{n}$, then $\alpha^{-1} \circ f \circ \alpha \in \mathfrak{P}_{n}$.

(c) Let $f \in \mathfrak{P}_{n}$. Then $f\left(E_{\mathbb{\boxplus}_{n}}(\infty, R)\right) \subset E_{\mathbb{\boxplus}_{n}}(\infty, R)$ for every $R>0$.

(d) Let $f \in \mathfrak{P}_{n}$ and write $f(z)=z+H(z)$ with $H=\left(H_{1}, \tilde{H}\right) \in \mathcal{K}\left(\mathbb{H}_{n}, c\right)$. Then

$$
\|\tilde{H}(z)\|^{2} \leq\left|H_{1}(z)-2 i \overline{\tilde{z}}^{T} \tilde{H}\right| \quad \text { for all } z=\left(z_{1}, \tilde{z}\right) \in \mathbb{U}_{n} .
$$

(e) Let $f \in \mathfrak{P}_{n}$. Then there exists $R>0$ such that $E_{\mathbb{H}_{n}}(\infty, R) \subset f\left(\mathbb{M}_{n}\right)$.

Proof. The statements (a) and (b) can easily be shown by using the explicit form of automorphisms of $\mathbb{M}_{n}$; see [Abate 1989, Proposition 2.2.4].

The statement (c) is just Julia's lemma: Write $f(z)=z+H(z)$ and let us pass to the unit ball and define $\tilde{f}: \mathbb{B}_{n} \rightarrow \mathbb{B}_{n}, \tilde{f}=C \circ f \circ C^{-1}$. Then

$$
\tilde{f}=\frac{1}{2 i+H_{1}\left(C^{-1}(z)\right)-z_{1} H_{1}\left(C^{-1}(z)\right)}\left(\left(\begin{array}{c}
\left(1-z_{1}\right) H_{1}\left(C^{-1}(z)\right) \\
2\left(1-z_{1}\right) \tilde{H}\left(C^{-1}(z)\right)
\end{array}\right)+2 i z\right) .
$$

By taking the sequence $z_{n}=(1-1 / n, 0)$, it is easy to see that

$$
\lim _{n \rightarrow \infty} \tilde{f}\left(z_{n}\right)=e_{1} \quad \text { and } \quad \lim _{n \rightarrow \infty} \frac{1-\left\|\tilde{f}\left(z_{n}\right)\right\|}{1-\left\|z_{n}\right\|}=1,
$$

i.e., $e_{1}$ is a boundary regular fixed point of $\tilde{f}$ with boundary dilatation coefficient $\leq 1$. Julia's lemma (see [Abate 1989, Theorem 2.2.21]) implies that $\tilde{f}\left(E_{\mathbb{B}_{n}}\left(e_{1}, R\right)\right) \subset$ $E_{\mathbb{B}_{n}}\left(e_{1}, R\right)$ for any $R>0$.

Inequality (d) follows directly from (c): Let $z=\left(z_{1}, \tilde{z}\right) \in \mathbb{U}_{n}$. Another formulation

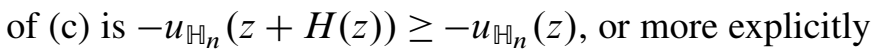

$$
\begin{aligned}
\operatorname{Im}\left(z_{1}\right)+\operatorname{Im}( & \left.H_{1}(z)\right)-\|\tilde{z}+\tilde{H}(z)\|^{2} \geq \operatorname{Im}\left(z_{1}\right)-\|\tilde{z}\|^{2} \\
& \Longleftrightarrow \operatorname{Im}\left(H_{1}(z)\right) \geq\|\tilde{z}+\tilde{H}(z)\|^{2}-\|\tilde{z}\|^{2}=2 \operatorname{Re}\left(\overline{\tilde{z}}^{T} \tilde{H}(z)\right)+\|\tilde{H}(z)\|^{2} \\
& \Longleftrightarrow \operatorname{Im}\left(H_{1}(z)-2 i \overline{\tilde{z}}^{T} \tilde{H}(z)\right) \geq\|\tilde{H}(z)\|^{2} .
\end{aligned}
$$

From this inequality it follows that $\|\tilde{H}(z)\|^{2} \leq\left|H_{1}(z)-2 i \overline{\tilde{z}}^{T} \tilde{H}\right|$ for all $z \in \mathbb{H}_{n}$. Finally we prove (e):

Let $f \in \mathfrak{P}_{n}$ and write $f(z)=z+H(z)$ with $H \in \mathcal{K}\left(\mathbb{H}_{n}, c\right)$. Because of (c), $f$ maps the horosphere $E_{\mathbb{H}_{n}}(\infty, 1)$ into itself. Hence the statement is proven if we

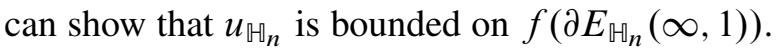

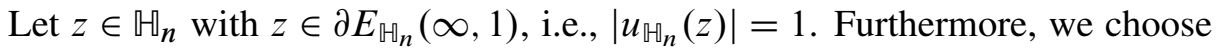
$\zeta \in \mathbb{U}$ and $\gamma \in \mathbb{C}$ such that $\varphi_{\gamma}(\zeta)=z$. Note that this implies $\left|u_{\mathbb{}}(\zeta)\right|=\operatorname{Im}(\zeta)=1$.

Let $P$ be the projection onto $\varphi_{\gamma}(\mathbb{M})$. 
Then we have

$$
\left|u_{\boxplus_{n}}(f(z))\right|=\left|u_{\boxplus_{n}}(z+H(z))\right|=\left|u_{\boxplus_{n}}(\underbrace{z+d P(z) H(z)}_{=: w}+\underbrace{H(z)-d P(z) H(z)}_{=: v})\right| .
$$

As $d P(z) \cdot d P(z)=d P(z)$, we have $d P(z) \cdot v=0$. A small calculation (see also [Casavecchia 2010, Lemma 3.1]) gives $v \in T_{z}^{\mathbb{C}} \partial E_{\mathbb{H}_{n}}(\infty, 1)$. Furthermore, also $w \in \varphi_{\gamma}(\mathbb{M})$ and $d P(z)=d P(w)$ and we get $v \in T_{w}^{\mathbb{C}} \partial E_{\mathbb{H}_{n}}\left(\infty,\left|u_{\boxplus_{n}}(w)\right|^{-1}\right)$. As

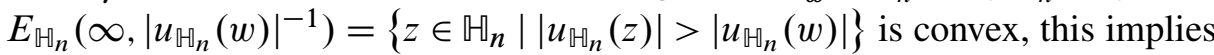

$$
\begin{aligned}
\left|u_{\uplus_{n}}(w+v)\right| \leq\left|u_{\uplus_{n}}(w)\right| & =\left|u_{\uplus_{n}}(z+d P(z) H(z))\right| \underset{\operatorname{Lemma} 3.3}{=}\left|u_{\uplus_{n}}\left(z+\left(h_{\gamma}(\zeta), 0\right)\right)\right| \\
& =\operatorname{Im}\left(z_{1}\right)-\|\tilde{z}\|^{2}+\operatorname{Im}\left(h_{\gamma}(\zeta)\right) \leq \operatorname{Im}\left(z_{1}\right)-\|\tilde{z}\|^{2}+\left|h_{\gamma}(\zeta)\right| \\
& =\left|u_{\uplus_{n}}(z)\right|+\left|h_{\gamma}(\zeta)\right|=1+\left|h_{\gamma}(\zeta)\right| \leq 1+\frac{c}{\operatorname{Im}(\zeta)}=1+c .
\end{aligned}
$$

Consequently, $f\left(\mathbb{\boxplus}_{n}\right) \supset f\left(E_{\mathbb{\sharp}}(\infty, 1)\right) \supset E_{\mathbb{H}_{n}}(\infty, 1+c)$.

Theorem 4.4. $\mathfrak{P}_{n}$ is a semigroup: if $f, g \in \mathfrak{P}_{n}$, then $f \circ g \in \mathfrak{P}_{n}$.

Proof. Let $f, g \in \mathfrak{P}_{n}$ with $F=\left(F_{1}, \widetilde{F}\right):=f-\mathrm{id}, G=\left(G_{1}, \widetilde{G}\right):=g-\mathrm{id}$ and

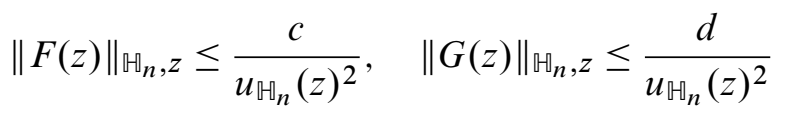

for all $z \in \mathbb{H}_{n}$. Let $z=\left(z_{1}, \tilde{z}\right) \in \mathbb{H}_{n}$ and $p=\left(p_{1}, \tilde{p}\right):=z+G(z)$.

From Remark 4.2, we know that $f \circ g-$ id is an infinitesimal generator on $\mathbb{H}_{n}$. It remains to estimate the hyperbolic metric of this generator. We have

$$
\begin{aligned}
& \|(f \circ g)(z)-z\|_{\mathbb{\boxplus}_{n}, z}=\|G(z)+F(z+G(z))\|_{\mathbb{\boxplus}_{n}, z}
\end{aligned}
$$

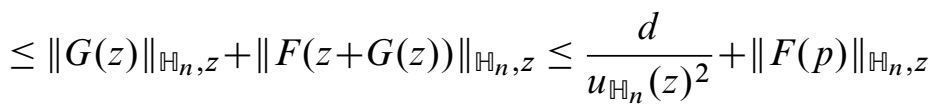

$$
\begin{aligned}
& \leq \frac{d}{u_{\uplus_{n}}(z)^{2}}+\left\|\left(F_{1}(p)-2 i \overline{\tilde{p}}^{T} \tilde{F}(p), 0\right)\right\|_{\mathbb{\boxplus}_{n}, z}+\left\|\left(2 i \overline{\tilde{p}}^{T} \tilde{F}(p), \tilde{F}(p)\right)\right\|_{\uplus_{n}, z} .
\end{aligned}
$$

Note that $F_{1}(p)-2 i \overline{\tilde{p}}^{T} \widetilde{F}(p)$ corresponds to the slice of $F$ with respect to the geodesic through $p$ and infinity. Because of Proposition 3.4, we know that

$$
\left|F_{1}(p)-2 i \overline{\tilde{p}}^{T} \tilde{F}(p)\right| \leq \frac{c}{\left|u_{\uplus_{n}}(p)\right|} \leq \frac{c}{\left|u_{\uplus_{n}}(z)\right|},
$$

where the second inequality follows from Proposition 4.3 (c). Together with (3-4), this implies

$$
\left\|\left(F_{1}(p)-2 i \overline{\tilde{p}}^{T} \tilde{F}(p), 0\right)\right\|_{\mathbb{\boxplus}_{n}, z}=\frac{\mid\left(F_{1}(p)-2 i \overline{\tilde{p}}^{T} \tilde{F}(p) \mid\right.}{\left|u_{\uplus_{n}}(z)\right|} \leq \frac{c}{u_{\uplus_{n}}(z)^{2}} .
$$


It remains to show that there exists a constant $C>0$ such that

$$
\left\|\left(2 i \overline{\tilde{p}}^{T} \tilde{F}(p), \tilde{F}(p)\right)\right\|_{\mathbb{\boxplus}_{n}, z} \leq \frac{C}{u_{\uplus_{n}}(z)^{2}} .
$$

First, (3-5) gives

$$
\begin{aligned}
& \left\|\left(2 i \overline{\tilde{p}}^{T} \widetilde{F}(p), \tilde{F}(p)\right)\right\|_{\uplus_{n}, z}=2 \frac{\sqrt{\left.\|\widetilde{F}(p)\|^{2}\left|u_{\boxplus_{n}}(z)\right|+\mid \overline{(\tilde{p}-\tilde{z}}\right)\left.^{T} \tilde{F}(p)\right|^{2}}}{\left|u_{\uplus_{n}}(z)\right|} \\
& \leq 2 \frac{\sqrt{\|\tilde{F}(p)\|^{2}\left|u_{\uplus_{n}}(z)\right|+\|(\tilde{p}-\tilde{z})\|^{2} \cdot\|\tilde{F}(p)\|^{2}}}{\left|u_{\uplus_{n}}(z)\right|}
\end{aligned}
$$

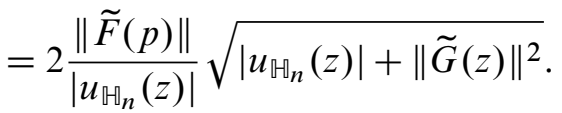

Now we differentiate between two cases.

Case 1: $\left|u_{\sharp_{n}}(z)\right| \geq 1$. The equations (3-8) and (3-10) imply

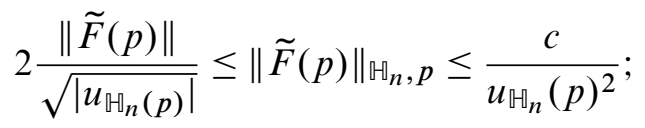

thus

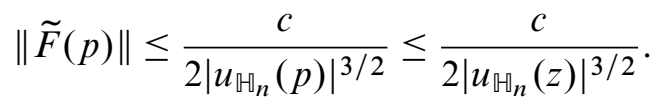

In the same way, we get

$$
\|\widetilde{G}(z)\| \leq \frac{d}{2\left|u_{\sharp_{n}}(z)\right|^{3 / 2}} .
$$

Combining (4-4) with (4-3) gives

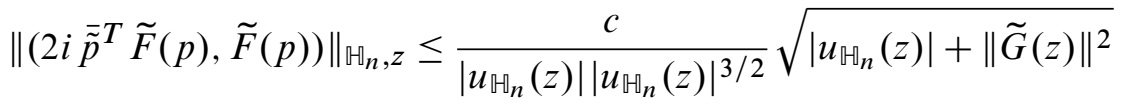

$$
\begin{aligned}
& =\frac{c}{\left|u_{\sharp_{n}}(z)\right|^{2}} \sqrt{1+\frac{\|\widetilde{G}(z)\|^{2}}{\left|u_{\boxplus_{n}}(z)\right|}} \\
& \leq \frac{c}{(4-5)} \frac{\left.u_{\boxplus_{n}}(z)\right|^{2}}{1+\frac{d^{2}}{4\left|u_{\boxplus_{n}}(z)\right|^{4}}} \\
& \leq \frac{c \sqrt{1+d^{2} / 4}}{\left|u_{\boxplus_{n}}(z)\right|^{2}} \text {. }
\end{aligned}
$$




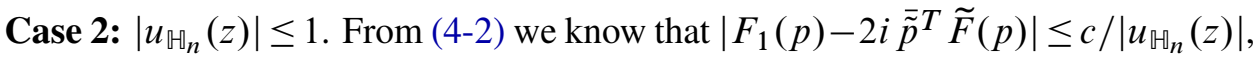
and (4-1) implies

$$
\|\widetilde{F}(p)\| \leq \frac{\sqrt{c}}{\sqrt{\left|u_{\uplus_{n}}(z)\right|}} .
$$

Similarly we get

$$
\|\widetilde{G}(z)\| \leq \frac{\sqrt{d}}{\sqrt{\left|\mathcal{u}_{\boxplus_{n}}(z)\right|}} .
$$

Hence, with (4-3) we obtain

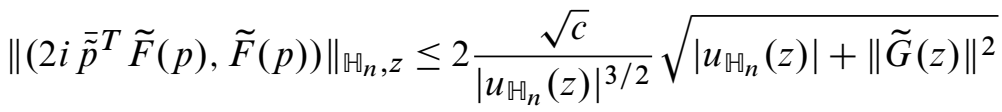

$$
\begin{aligned}
& \leq 2 \frac{\sqrt{c}}{\left|u_{\boxplus_{n}}(z)\right|^{3 / 2}} \sqrt{\left|u_{\boxplus_{n}}(z)\right|+\frac{d}{\left|u_{\boxplus_{n}}(z)\right|}} \\
& =2 \frac{\sqrt{c}}{\left|u_{\uplus_{n}}(z)\right|^{2}} \sqrt{u_{\boxplus_{n}}(z)^{2}+d} \\
& \leq 2 \frac{\sqrt{c}}{\left|u_{\mathbb{\boxplus}_{n}}(z)\right|^{2}} \sqrt{1+d} \text {. }
\end{aligned}
$$

On the Loewner equation with a $\mathcal{K}\left(\mathbb{W}_{n}, \boldsymbol{c}\right)$-Herglotz vector field. Let $\left\{\Phi_{t}\right\}_{t \geq 0}$ be a semigroup on $\mathbb{H}_{n}$ with generator $H \in \mathcal{K}\left(\mathbb{H}_{n}, c\right)$. Next we will show that this implies $\Phi_{t} \in \mathfrak{P}_{n}$ for every $t \geq 0$.

In fact we can prove a little more by considering a nonautonomous version of (1-1). To this end, let $\left\{H_{t}: \mathbb{W}_{n} \rightarrow \mathbb{C}^{n}\right\}_{t \geq 0}$ be a $\mathcal{K}\left(\mathbb{W}_{n}, c\right)$-Herglotz vector field, i.e., $H_{t} \in \mathcal{K}\left(\mathbb{H}_{n}, c\right)$ for almost every $t \geq 0$ and the map $t \mapsto H_{t}(z)$ is measurable for every $z \in \mathbb{M}_{n}$; see [Arosio and Bracci 2011, Definition 1.2]. In this case, one can solve the nonautonomous version of (1-1), namely the Loewner equation

$$
\frac{\partial \varphi_{t}(z)}{\partial t}=H_{t}\left(\varphi_{t}(z)\right), \quad \varphi_{0}(z)=z \in \mathbb{H}_{n},
$$

which gives a family $\left\{\varphi_{t}\right\}_{t \geq 0}$ of univalent self-mappings of $\mathbb{U}_{n}$; see [Arosio and Bracci 2011, Theorem 1.4].

Theorem 4.5. If $\left\{H_{t}\right\}_{t \geq 0}$ is a $\mathcal{K}\left(\mathbb{U}_{n}, c\right)$-Herglotz vector field and $\left\{\varphi_{t}\right\}_{t \geq 0}$ the solution to (4-6), then $\varphi_{t} \in \mathfrak{P}_{n}$ for every $t \geq 0$.

Proof. Firstly, for every $t \geq 0$ and $R>0$, the map $\varphi_{t}$ maps the horosphere $E_{\mathbb{\forall} \sharp_{n}}(\infty, R)$ into itself, i.e.,

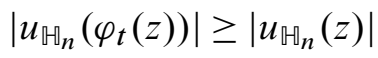

for every $z \in \mathbb{H}_{n}$. This can be seen as follows: 
First, consider the autonomous case $H_{t}(z)=J(z)$ for every $t \geq 0$ and some $J \in \mathcal{K}\left(\mathbb{\boxplus}_{n}, c\right)$. Let $G$ be the corresponding generator in the unit ball, i.e., $G=$ $C^{\prime}\left(C^{-1}\right) \cdot\left(J \circ C^{-1}\right)$. Then $G$ satisfies the inequality

$$
\|G(z)\| \leq\|G(z)\|_{\mathbb{B}_{n}, z} \leq \frac{c}{u_{\mathbb{B}_{n}}(z)^{2}}=\frac{c\left|1-z_{1}\right|^{4}}{\left(1-\|z\|^{2}\right)^{2}} .
$$

Putting $z=r \cdot e_{1}$ gives

$$
\left\|G\left(r e_{1}\right)\right\| \leq \frac{c(1-r)^{4}}{\left(1-r^{2}\right)^{2}}=\frac{c(1-r)^{2}}{(1+r)^{2}} .
$$

From this it follows immediately that

$$
\lim _{(0,1) \ni r \rightarrow 1} G\left(r e_{1}\right)=0 \quad \text { and } \quad \lim _{(0,1) \ni r \rightarrow 1} \frac{G_{1}\left(r e_{1}\right)}{r-1}=0 .
$$

Theorem 0.3 in [Bracci et al. 2010] implies that $e_{1}$ is a boundary regular fixed point for the generated semigroup with boundary dilatation coefficient 1 . Hence we can apply Julia's lemma and obtain (4-7).

Now assume that $H_{t}(z)$ is piecewise constant with respect to time. By using the previous case, we see that (4-7) also holds in this case.

Finally, for a general $\mathcal{K}\left(\mathbb{H}_{n}, c\right)$-Herglotz vector field $H_{t}(z)$, we can approximate the solution $\varphi_{t}$ by a sequence $\varphi_{t, n}$ such that for each $n$, the family $\left\{\varphi_{t, n}\right\}_{t \geq 0}$ solves (4-6) with a piecewise constant $\mathcal{K}\left(\mathbb{H}_{n}, c\right)$-Herglotz vector field. By using the continuity of $u_{\sharp_{n}}(z)$, we see that (4-7) also holds for $\varphi_{t}$.

Let $z=\left(z_{1}, z_{2}\right) \in \mathbb{H}_{n}$ and write $\varphi_{t}=\left(\varphi_{1, t}, \tilde{\varphi}_{t}\right), H_{t}=\left(H_{1, t}, \tilde{H}_{t}\right)$. The mapping $\varphi_{t}$ satisfies the integral equation

$$
\varphi_{t}(z)=z+\int_{0}^{t} H_{s}\left(\varphi_{s}(z)\right) d s .
$$

Similarly to the proof of Theorem 4.4, (4-4), we deduce from the fact that $H_{t} \in$ $\mathcal{K}\left(\mathbb{H}_{n}, c\right)$ for almost every $t \geq 0$ and equations (3-8) and (3-10) that

$$
\left\|\tilde{H}_{t}\left(\varphi_{t}(z)\right)\right\| \leq \frac{c}{2\left|u_{\boxplus_{n}}(z)\right|^{3 / 2}}
$$

for every $z \in \mathbb{M}_{n}$ and almost every $t \geq 0$, and similarly to (4-2), we deduce that

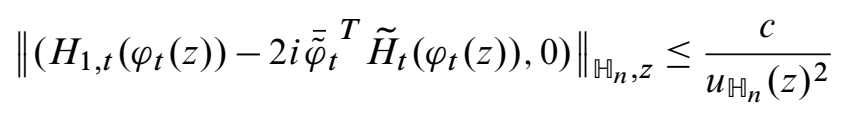

for every $z \in \mathbb{H}_{n}$ and almost every $t \geq 0$.

First we get

(4-10) $\left\|\tilde{\varphi}_{s}-\tilde{z}\right\| \leq \int_{0}^{s}\left\|\tilde{H}_{\tau}\left(\varphi_{\tau}(z)\right)\right\| d \tau \leq \int_{0}^{s} \frac{c}{2\left|u_{\sharp_{n}}(z)\right|^{3 / 2}} d \tau=\frac{c s}{2\left|u_{\uplus_{n}}(z)\right|^{3 / 2}}$. 
Suppose $\left|u_{\varpi_{n}}(z)\right| \geq 1$. Then we have

$$
\begin{aligned}
& \left\|\varphi_{t}(z)-z\right\|_{\mathbb{H}_{n}, z} \leq \int_{0}^{t}\left\|H_{S}\left(\varphi_{s}(z)\right)\right\|_{\mathbb{H}_{n}, z} d s \\
& \leq \int_{0}^{t}\left\|\left(\begin{array}{c}
H_{1, s}\left(\varphi_{s}(z)\right)-2 i \overline{\tilde{\varphi}}_{s}^{T} \tilde{H}_{S}\left(\varphi_{s}(z)\right) \\
0
\end{array}\right)\right\|_{\mathbb{Q}_{n}, z} d s \\
& +\int_{0}^{t}\left\|\left(\begin{array}{c}
2 i \overline{\tilde{\varphi}}_{s}^{T} \tilde{H}_{S}\left(\varphi_{s}(z)\right) \\
\tilde{H}_{S}\left(\varphi_{S}(z)\right)
\end{array}\right)\right\|_{\mathbb{H}_{n}, z} d s
\end{aligned}
$$

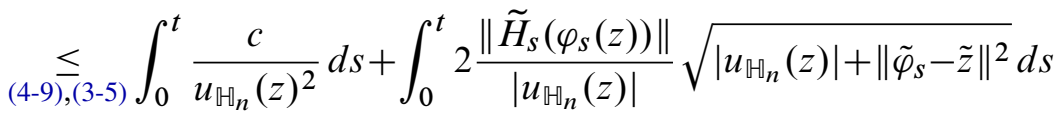

$$
\begin{aligned}
& \underset{(4-8),(4-10)}{\leq} \int_{0}^{t} \frac{c}{u_{\boxplus_{n}}(z)^{2}} d s+\int_{0}^{t} \frac{c}{\left|u_{\boxplus_{n}}(z)\right|^{5 / 2}} \sqrt{\left|u_{\uplus_{n}}(z)\right|+\frac{c^{2} s^{2}}{4\left|u_{\boxplus_{n}}(z)\right|^{3}}} d s
\end{aligned}
$$

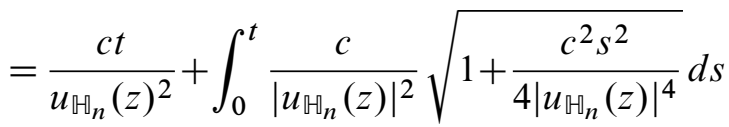

$$
\begin{aligned}
& \leq \frac{c t}{u_{\uplus_{n}}(z)^{2}}+\int_{0}^{t} \frac{c}{\left|u_{\uplus_{n}}(z)\right|^{2}} \sqrt{1+c^{2} s^{2}} d s
\end{aligned}
$$

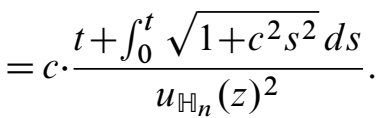

The case $\left|u_{\sharp_{n}}(z)\right| \leq 1$ is treated similarly, compare with the proof of Theorem 4.4, and we conclude that for every $t \geq 0$, there exists $C>0$ such that $\left\|\varphi_{t}(z)-z\right\|_{\mathbb{H}_{n}} \leq$ $C / u_{\mathbb{H}_{n}}(z)^{2}$ for all $z \in \mathbb{\boxplus}_{n}$. Together with Remark 4.2, this implies that $\varphi_{t} \in \mathfrak{P}_{n}$.

Question 4.6. Let $f \in \mathfrak{P}_{1}$. In [Goryaĭnov and Ba 1992, Section 4], it is shown that there exists a $\mathcal{K}(\mathbb{H}, c)$-Herglotz vector field $H_{t}$ and a time $T \geq 0$ such that $f=\varphi_{T}$, where $\left\{\varphi_{t}\right\}_{t \geq 0}$ is the solution of (4-6). What can be said in the higher-dimensional case?

On the behavior of iterates. Let $F: \mathbb{B}_{n} \rightarrow \mathbb{B}_{n}$ be holomorphic. We say that $p \in \overline{\mathbb{B}}_{n}$ is the Denjoy-Wolff point of $F$ if $F^{n} \rightarrow p$ for $n \rightarrow \infty$ locally uniformly. The basic results about the behavior of the iterates $F^{n}$ for $n \rightarrow \infty$ can be found in [Abate 1989, Chapter 2.2]. In particular we have (Theorem 2.2.31)

$F$ has a Denjoy-Wolff point on the boundary $\partial \mathbb{B}_{n} \Longleftrightarrow F$ has no fixed points.

Now let $f \in \mathfrak{P}_{n}$. For $n=1, f$ has the Denjoy-Wolff point $\infty$ if $f$ is not the identity: As $f$ is not an elliptic automorphism, the classical Denjoy-Wolff theorem 
implies that $f$ has a Denjoy-Wolff point. This point has to be $\infty$, e.g., because of Proposition 4.3 (c).

Next we will show that this is also true in higher dimensions, provided that $f$ extends smoothly to the boundary point $\infty$. There are different possible definitions of smoothness of $f$ near $\infty$. We will use the following one: Let $H(z)=f(z)-z$, and denote by $G: \mathbb{B}_{n} \rightarrow \mathbb{C}^{n}$ the corresponding generator on $\mathbb{B}_{n}$; i.e., we have

$$
H(z)=\left(C^{-1}\right)^{\prime}(C(z)) \cdot G(C(z))
$$

and a small computation shows

$$
H_{1}(z)=-\frac{i}{2}\left(z_{1}+i\right)^{2} \cdot G_{1}(C(z)) .
$$

Our smoothness condition will be that $G_{1}$ has a $C^{3}$-extension to $e_{1}$; i.e., we can write

$$
G_{1}(z)=\sum_{\substack{k_{1}+\cdots+k_{n} \leq 3 \\ k_{1}, \ldots, k_{n} \geq 0}} a_{k_{1}, \ldots, k_{n}}\left(z_{1}-1\right)^{k_{1}} \cdot z_{2}^{k_{2}} \cdots \cdots z_{n}^{k_{n}}+\mathcal{O}\left(\left\|z-e_{1}\right\|^{3}\right),
$$

which translates to

$H_{1}(z)=-\frac{i}{2}\left(z_{1}+i\right)^{2}$.

$\sum_{k_{1}+\cdots+k_{n} \leq 3} a_{k_{1}, \ldots, k_{n}}\left(\frac{-2 i}{z_{1}+i}\right)^{k_{1}} \cdot\left(\frac{2 z_{2}}{z_{1}+i}\right)^{k_{2}} \cdots\left(\frac{2 z_{n}}{z_{1}+i}\right)^{k_{n}}+\mathcal{O}\left(\left\|C(z)-e_{1}\right\|^{3}\right)$,

or

$$
\begin{aligned}
& H_{1}(z)=b_{0, \ldots, 0} \cdot\left(z_{1}+i\right)^{2}+\left(z_{1}+i\right) \cdot \sum_{k_{1}+\cdots+k_{n}=1} b_{k_{1}, \ldots, k_{n}} z_{2}^{k_{2} \cdots \cdots z_{n}^{k_{n}}}
\end{aligned}
$$

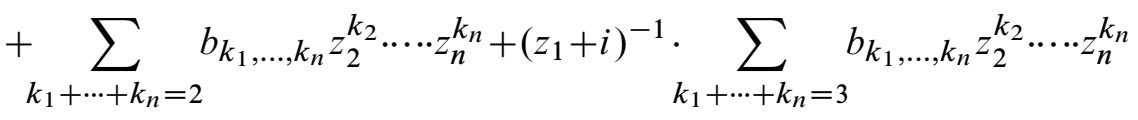

$$
\begin{aligned}
& +\mathcal{O}\left(\left|z_{1}+i\right|^{-1} \cdot\left\|\left(1, z_{2}, \ldots, z_{n}\right)\right\|^{3}\right)
\end{aligned}
$$

for some coefficients $b_{k_{1}, \ldots, k_{n}} \in \mathbb{C}$.

Theorem 4.7. Let $f \in \mathfrak{P}_{n}, f \neq \mathrm{id}$, and assume that (4-12) is satisfied. Then $\infty$ is the Denjoy-Wolff point of $f$.

Proof. Write $f(z)=z+H(z)$, where $H \in \mathcal{K}\left(\mathbb{H}_{n}, c\right)$ and $H=\left(H_{1}, \widetilde{H}\right)$. Let $\gamma \in \mathbb{C}^{n-1}$. If we can show that the slice $h_{\gamma}(\zeta)=H_{1}(\varphi(\zeta))-2 i \bar{\gamma}^{T} \tilde{H}\left(\varphi_{\gamma}(\zeta)\right)$ has no zeros, then we are done: 
This implies that $H$ has no zeros because of (3-7) and (3-8). Hence, $f$ has no fixed points and (4-11) implies that $f$ has a Denjoy-Wolff point. This point has to be $\infty$ because of Proposition 4.3 (c).

Similarly to the proof of Theorem 4.4, (4-4), we have

$$
\|\tilde{H}(z)\| \leq \frac{c}{2\left|u_{\uplus_{n}}(z)\right|^{3 / 2}},
$$

and thus

$$
\left\|\tilde{H}\left(\varphi_{\gamma}(\zeta)\right)\right\| \leq \frac{c}{2\left|u_{\boxplus_{n}}\left(\varphi_{\gamma}(\zeta)\right)\right|^{3 / 2}}=\frac{c}{2 \operatorname{Im}(\zeta)^{3 / 2}}
$$

Consequently,

$$
\lim _{y \rightarrow \infty} y\left|\bar{\gamma}^{T} \tilde{H}\left(\varphi_{\gamma}(i y)\right)\right|=0 .
$$

On the other hand, we know from Proposition 3.4 that $h_{\gamma} \in \mathcal{K}(\mathbb{H}, c)$, which implies (see Remark 1.5)

$$
\limsup _{y \rightarrow \infty} y\left|h_{\gamma}(i y)\right|=\limsup _{y \rightarrow \infty} y\left|H_{1}(\varphi(i y))-2 i \bar{\gamma}^{T} \tilde{H}\left(\varphi_{\gamma}(i y)\right)\right| \leq c
$$

which gives us

$$
\limsup _{y \rightarrow \infty}\left|i y \cdot H_{1}\left(\varphi_{\gamma}(i y)\right)\right| \leq c
$$

Now we use the assumption of the smoothness of $H_{1}$ :

Because of (4-13), all coefficients $b_{k_{1}, \ldots, k_{n}}$ from (4-12) with $k_{1}+\cdots+k_{n} \leq 2$ have to be 0 . Thus,

$$
\lim _{y \rightarrow \infty} i y \cdot H_{1}\left(\varphi_{\gamma}(i y)\right)=: K(\gamma)
$$

exists and is a polynomial in $\gamma=\left(\gamma_{2}, \ldots, \gamma_{n}\right)$ :

$$
K(\gamma)=\sum_{k_{1}+\cdots+k_{n}=3} b_{k_{1}, \ldots, k_{n}} \gamma_{2}^{k_{2}} \cdots \cdots \gamma_{n}^{k_{n}} .
$$

As $K(\gamma)$ is bounded, it has to be constant.

If $K(\gamma) \equiv 0$, then all slices of $H$ are zero; hence $H=0$ by Remark 3.5 and $f$ is the identity, a contradiction.

Hence $K(\gamma)$ is a nonzero constant and $h_{\gamma}(\zeta)$ is not identically zero, which implies (e.g., by using the representation (1-5)) that $h_{\gamma}(\zeta)$ has no zeros.

Question 4.8. Is $\infty$ the Denjoy-Wolff point for every $f \in \mathfrak{P}_{n}$ ? 


\section{Appendix: Proof of Lemma 3.2}

Lemma 3.2. Let $a \in \mathbb{C}, p, v \in \mathbb{C}^{n-1}$ and $z=\left(z_{1}, \tilde{z}\right) \in \mathbb{H}_{n}$. Then the following formulas hold:

$$
\begin{aligned}
& \left\|\left(\begin{array}{l}
a \\
0
\end{array}\right)\right\|_{\mathbb{H}_{n}, z}=\frac{|a|}{\left|u_{\mathbb{H}_{n}}(z)\right|},
\end{aligned}
$$

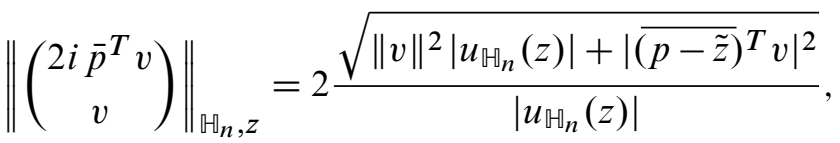

$$
\left\|\left(\begin{array}{c}
a-2 i \overline{\tilde{z}}^{T} v \\
0
\end{array}\right)+\left(\begin{array}{c}
2 i \overline{\tilde{z}}^{T} v \\
v
\end{array}\right)\right\|_{\mathbb{H}_{n}, z}^{2}=\left\|\left(\begin{array}{c}
a-2 i \overline{\tilde{z}}^{T} v \\
0
\end{array}\right)\right\|_{\mathbb{H}_{n}, z}^{2}+\left\|\left(\begin{array}{c}
2 i \overline{\tilde{z}}^{T} v \\
v
\end{array}\right)\right\|_{\mathbb{H}_{n}, z}^{2} .
$$

Proof. We write $\tilde{z}=\left(z_{2}, \ldots, z_{n}\right), v=\left(v_{2}, \ldots, v_{n}\right), p=\left(p_{2}, \ldots, p_{n}\right)$.

An explicit formula of the Kobayashi metric for the unit ball is given in [Abate 2004, Theorem 3.4]. ${ }^{4}$ It coincides with the Bergman metric and by using the Cayley map, we get the following formula for the upper half-space:

$$
\|w\|_{\mathbb{H}_{n}, z}^{2}=w^{T} \cdot\left(g_{j, k}\right)_{j, k} \cdot \bar{w},
$$

where $w \in \mathbb{C}^{n}$ and $\left(g_{j, k}\right)_{j, k}$ is an $n \times n$-matrix with

$$
g_{j, k}=-4 \frac{\partial^{2}}{\partial z_{j} \partial \bar{z}_{k}} \log \left(\operatorname{Im}\left(z_{1}\right)-\sum_{l=2}^{n}\left|z_{l}\right|^{2}\right),
$$

and we get for $j, k \geq 2$,

$$
\begin{aligned}
& g_{1,1}=\frac{1}{u_{\boxplus_{n}}(z)^{2}}, \quad g_{1, k}=\frac{2 i z_{k}}{u_{\boxplus_{n}}(z)^{2}}, \quad g_{j, 1}=\frac{-2 i \bar{z}_{j}}{u_{\boxplus_{n}}(z)^{2}},
\end{aligned}
$$

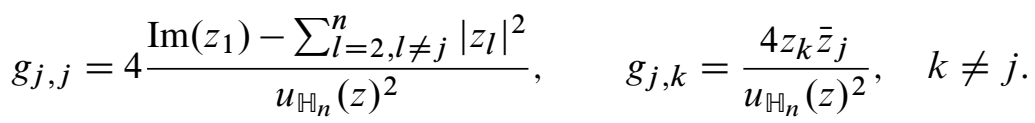

The formulas (3-4) and (3-5) are now straightforward calculations. We obtain

$$
\|(a, 0)\|_{\mathbb{\boxplus}_{n}, z}=\sqrt{(a, 0) \cdot\left(g_{j, k}\right)_{j, k} \cdot \overline{(a, 0)^{T}}}=\sqrt{a \cdot g_{1,1} \cdot \bar{a}}=\frac{|a|}{\left|u_{\sharp_{n}}(z)\right|},
$$

${ }^{4}$ Note, however, that the Kobayashi metric in [Abate 2004] differs by a factor of 2 from the one we are using here. 
and

$$
\begin{aligned}
& u_{\mathbb{\boxplus}_{n}}(z)^{2} \cdot\left\|\left(2 i \bar{p}^{T} v, v\right)\right\|_{\mathbb{\boxplus}_{n}, z}^{2}
\end{aligned}
$$

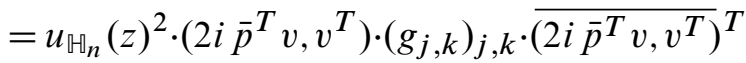

$$
\begin{aligned}
& =u_{\varpi_{n}}(z)^{2} \cdot\left(\sum_{j=2}^{n} g_{j, j}\left|v_{j}\right|^{2}+g_{1,1}\left|2 i \bar{p}^{T} v\right|^{2}\right. \\
& \left.+\sum_{j=2}^{n} g_{j, 1} v_{j} \overline{2 i \bar{p}^{T} v}+\sum_{k=2}^{n} g_{1, k} \bar{v}_{j} 2 i \bar{p}^{T} v+\sum_{j, k \geq 2, j \neq k}^{n} g_{j, k} v_{j} \bar{v}_{k}\right) \\
& =4 \sum_{j=2}^{n}\left(\operatorname{Im}\left(z_{1}\right)-\|\tilde{z}\|^{2}\right) \cdot\left|v_{j}\right|^{2}+4 \sum_{j=2}^{n}\left|z_{j}\right|^{2} \cdot\left|v_{j}\right|^{2}+4 \sum_{j, k \geq 2}^{n} p_{j} \bar{p}_{k} v_{j} \bar{v}_{k} \\
& -4 \sum_{j, k \geq 2}^{n} \bar{z}_{j} p_{k} v_{j} \bar{v}_{k}-4 \sum_{j, k \geq 2}^{n} z_{j} \bar{p}_{k} \bar{v}_{j} v_{k}+4 \sum_{j, k \geq 2, j \neq k}^{n} \bar{z}_{j} z_{k} v_{j} \bar{v}_{k} \\
& =4\|v\|^{2} \cdot\left|u_{\boxplus_{n}}(z)\right|+4 \sum_{j=2}^{n} z_{j} \bar{z}_{j} v_{j} \bar{z}_{j} \\
& +4 \sum_{j, k \geq 2}^{n}\left(p_{j} \bar{p}_{k} v_{j} \bar{v}_{k}-\bar{z}_{j} p_{k} v_{j} \bar{v}_{k}-z_{j} \bar{p}_{k} \bar{v}_{j} v_{k}\right)+4 \sum_{j, k \geq 2, j \neq k}^{n} \bar{z}_{j} z_{k} v_{j} \bar{v}_{k} \\
& =4\|v\|^{2} \cdot\left|u_{\uplus_{n}}(z)\right|+4 \sum_{j, k \geq 2}^{n}\left(p_{j} \bar{p}_{k} v_{j} \bar{v}_{k}-\bar{z}_{j} p_{k} v_{j} \bar{v}_{k}-z_{j} \bar{p}_{k} \bar{v}_{j} v_{k}+\bar{z}_{j} z_{k} v_{j} \bar{v}_{k}\right) \\
& =4\|v\|^{2} \cdot\left|u_{\varpi_{n}}(z)\right|+4\left|\overline{(p-\tilde{z})^{T}} v\right|^{2} .
\end{aligned}
$$

For formula (3-6) we just need to show that

$$
\left(2 i \overline{\tilde{z}}^{T} v, v^{T}\right) \cdot\left(g_{j, k}\right)_{j, k} \cdot{\overline{\left(a-2 i \overline{\tilde{z}}^{T} v, 0\right)}}^{T}=0 .
$$

Indeed, we have

$$
\begin{aligned}
& \left.u_{\sharp_{n}}(z)^{2} \cdot\left(g_{j, k}\right)_{j, k} \cdot \overline{\left(a-2 i \bar{z}^{T} v, 0\right)}\right)^{T} \\
& =\left(\bar{a}+2 i \tilde{z}^{T} \bar{v},-2 i \bar{z}_{2} \bar{a}+4 \bar{z}_{2} \tilde{z}^{T} \bar{v}, \ldots,-2 i \bar{z}_{n} \bar{a}+4 \bar{z}_{n} \tilde{z}^{T} \bar{v}\right)^{T}
\end{aligned}
$$

and

$$
\begin{aligned}
\left(2 i \overline{\tilde{z}}^{T} v, v^{T}\right)\left(\bar{a}+2 i \tilde{z}^{T} \bar{v},-2 i \bar{z}_{2} \bar{a}+4 \bar{z}_{2} \tilde{z}^{T} \bar{v}, \ldots,-2 i \bar{z}_{n} \bar{a}+4 \bar{z}_{n} \tilde{z}^{T} \bar{v}\right)^{T} & \\
= & 2 i \bar{a} \overline{\tilde{z}} \overline{\tilde{z}}^{T} v-4\left|\tilde{z}^{T} \bar{v}\right|^{2}-2 i \bar{a} \overline{\tilde{z}} T+4\left|\tilde{z}^{T} \bar{v}\right|^{2}=0 .
\end{aligned}
$$




\section{References}

[Abate 1989] M. Abate, Iteration theory of holomorphic maps on taut manifolds, Mediterranean Press, Rende, 1989. MR 92i:32032 Zbl 0747.32002

[Abate 1992] M. Abate, "The infinitesimal generators of semigroups of holomorphic maps", Ann. Mat. Pura Appl. (4) 161 (1992), 167-180. MR 93i:32029 Zbl 0758.32013

[Abate 2004] M. Abate, "Angular derivatives in several complex variables", pp. 1-47 in Real methods in complex and CR geometry (Martina Franca, 2002), Lecture Notes in Mathematics 1848, Springer, Berlin, 2004. MR 2005g:32019 Zbl 1068.32006

[Abate et al. 2010] M. Abate, F. Bracci, M. D. Contreras, and S. Díaz-Madrigal, "The evolution of Loewner's differential equations", Eur. Math. Soc. Newsl. 78 (2010), 31-38. MR 2768999 Zbl 1230.30003

[Arosio and Bracci 2011] L. Arosio and F. Bracci, "Infinitesimal generators and the Loewner equation on complete hyperbolic manifolds", Anal. Math. Phys. 1:4 (2011), 337-350. MR 2887104 Zbl 1254.32037

[Bauer 2005] R. O. Bauer, "Chordal Loewner families and univalent Cauchy transforms", J. Math. Anal. Appl. 302:2 (2005), 484-501. MR 2005g:30009 Zbl 1067.30035

[Berkson and Porta 1978] E. Berkson and H. Porta, "Semigroups of analytic functions and composition operators”, Michigan Math. J. 25:1 (1978), 101-115. MR 58 \#1112 Zbl 0382.47017

[Bracci and Patrizio 2005] F. Bracci and G. Patrizio, "Monge-Ampère foliations with singularities at the boundary of strongly convex domains", Math. Ann. 332:3 (2005), 499-522. MR 2006j:32048 Zbl 1086.32028

[Bracci and Shoikhet 2014] F. Bracci and D. Shoikhet, "Boundary behavior of infinitesimal generators in the unit ball”, Trans. Amer. Math. Soc. 366:2 (2014), 1119-1140. MR 3130328 Zbl 06265559

[Bracci et al. 2010] F. Bracci, M. D. Contreras, and S. Díaz-Madrigal, "Pluripotential theory, semigroups and boundary behavior of infinitesimal generators in strongly convex domains", J. Eur. Math. Soc. (JEMS) 12:1 (2010), 23-53. MR 2011c:32025 Zbl 1185.32010

[Bracci et al. 2014] F. Bracci, M. Elin, and D. Shoikhet, "Growth estimates for pseudo-dissipative holomorphic maps in Banach spaces", J. Nonlinear Convex Anal. 15:1 (2014), 191-198. MR 3184757 Zbl 1297.46033

[Casavecchia 2010] T. Casavecchia, "A rigidity condition for generators in strongly convex domains", Complex Var. Elliptic Equ. 55:12 (2010), 1131-1142. MR 2012e:32025 Zbl 1213.32010

[Contreras et al. 2010] M. D. Contreras, S. Díaz-Madrigal, and P. Gumenyuk, "Geometry behind chordal Loewner chains", Complex Anal. Oper. Theory 4:3 (2010), 541-587. MR 2011h:30037 Zbl 1209.30010

[Goryaŭnov and Ba 1992] V. V. Goryaĭnov and I. Ba, "Semigroup of conformal mappings of the upper half-plane into itself with hydrodynamic normalization at infinity", Ukraïn. Mat. Zh. 44:10 (1992), 1320-1329. In Russian; translated in Ukrainian Math. J. 44:10 (1992), 1209-1217. MR 94b:30013 Zbl 0873.30006

[Graham and Kohr 2003] I. Graham and G. Kohr, Geometric function theory in one and higher dimensions, Monographs and Textbooks in Pure and Applied Mathematics 255, Marcel Dekker, New York, 2003. MR 2004i:32002 Zbl 1042.30001

[Maassen 1992] H. Maassen, "Addition of freely independent random variables", J. Funct. Anal. 106:2 (1992), 409-438. MR 94g:46069 Zbl 0784.46047

[Pommerenke 1975] C. Pommerenke, Univalent functions, Vandenhoeck \& Ruprecht, Göttingen, 1975. MR 58 \#22526 Zbl 0298.30014 
[Poreda 1987a] T. Poreda, "On the univalent holomorphic maps of the unit polydisc in $\mathbf{C}^{n}$ which have the parametric representation, I: The geometrical properties", Ann. Univ. Mariae Curie-Skłodowska Sect. A 41 (1987), 105-113. MR 91m:32021 Zbl 0698.32004

[Poreda 1987b] T. Poreda, "On the univalent holomorphic maps of the unit polydisc in $\mathbf{C}^{n}$ which have the parametric representation, II: The necessary conditions and the sufficient conditions", Ann. Univ. Mariae Curie-Sktodowska Sect. A 41 (1987), 115-121. MR 91m:32022 Zbl 0698.32005

[Reich and Shoikhet 2005] S. Reich and D. Shoikhet, Nonlinear semigroups, fixed points, and geometry of domains in Banach spaces, Imperial College Press, London, 2005. MR 2006g:47105 Zbl 1089.46002

Received January 16, 2015. Revised May 4, 2015.

SEBASTIAN SCHLEISSINGER

DIPARTIMENTO DI MATEMATICA

UNIVERSITÀ DI ROMA TOR VERGATA

VIA DELLA RICERCA SCIENTIFICA 1

00133 ROMA

ITALY

sebastian.schleissinger@mathematik.uni-wuerzburg.de 


\title{
PACIFIC JOURNAL OF MATHEMATICS
}

\author{
msp.org/pjm
}

Founded in 1951 by E. F. Beckenbach (1906-1982) and F. Wolf (1904-1989)

\section{EDITORS}

Don Blasius (Managing Editor)

Department of Mathematics

University of California

Los Angeles, CA 90095-1555

blasius@math.ucla.edu

\author{
Paul Balmer \\ Department of Mathematics \\ University of California \\ Los Angeles, CA 90095-1555 \\ balmer@math.ucla.edu \\ Robert Finn \\ Department of Mathematics \\ Stanford University \\ Stanford, CA 94305-2125 \\ finn@math.stanford.edu \\ Sorin Popa \\ Department of Mathematics \\ University of California \\ Los Angeles, CA 90095-1555 \\ popa@math.ucla.edu
}

\author{
Vyjayanthi Chari \\ Department of Mathematics \\ University of California \\ Riverside, CA 92521-0135 \\ chari@math.ucr.edu \\ Kefeng Liu \\ Department of Mathematics \\ University of California \\ Los Angeles, CA 90095-1555 \\ liu@math.ucla.edu \\ Jie Qing \\ Department of Mathematics \\ University of California \\ Santa Cruz, CA 95064 \\ qing@ cats.ucsc.edu
}

\section{PRODUCTION}

Silvio Levy, Scientific Editor, production@msp.org

\section{SUPPORTING INSTITUTIONS}

ACADEMIA SINICA, TAIPEI

CALIFORNIA INST. OF TECHNOLOGY

INST. DE MATEMÁTICA PURA E APLICADA

KEIO UNIVERSITY

MATH. SCIENCES RESEARCH INSTITUTE

NEW MEXICO STATE UNIV.

OREGON STATE UNIV.

\author{
STANFORD UNIVERSITY \\ UNIV. OF BRITISH COLUMBIA \\ UNIV. OF CALIFORNIA, BERKELEY \\ UNIV. OF CALIFORNIA, DAVIS \\ UNIV. OF CALIFORNIA, LOS ANGELES \\ UNIV. OF CALIFORNIA, RIVERSIDE \\ UNIV. OF CALIFORNIA, SAN DIEGO \\ UNIV. OF CALIF., SANTA BARBARA
}

\author{
Daryl Cooper \\ Department of Mathematics \\ University of California \\ Santa Barbara, CA 93106-3080 \\ cooper@math.ucsb.edu \\ Jiang-Hua Lu \\ Department of Mathematics \\ The University of Hong Kong \\ Pokfulam Rd., Hong Kong \\ jhlu@maths.hku.hk \\ Paul Yang \\ Department of Mathematics \\ Princeton University \\ Princeton NJ 08544-1000 \\ yang@math.princeton.edu
}

These supporting institutions contribute to the cost of publication of this Journal, but they are not owners or publishers and have no responsibility for its contents or policies.

See inside back cover or msp.org/pjm for submission instructions.

The subscription price for 2016 is US $\$ 440 /$ year for the electronic version, and $\$ 600 /$ year for print and electronic.

Subscriptions, requests for back issues and changes of subscribers address should be sent to Pacific Journal of Mathematics, P.O. Box 4163, Berkeley, CA 94704-0163, U.S.A. The Pacific Journal of Mathematics is indexed by Mathematical Reviews, Zentralblatt MATH, PASCAL CNRS Index, Referativnyi Zhurnal, Current Mathematical Publications and Web of Knowledge (Science Citation Index).

The Pacific Journal of Mathematics (ISSN 0030-8730) at the University of California, c/o Department of Mathematics, 798 Evans Hall \#3840, Berkeley, CA 94720-3840, is published twelve times a year. Periodical rate postage paid at Berkeley, CA 94704, and additional mailing offices. POSTMASTER: send address changes to Pacific Journal of Mathematics, P.O. Box 4163, Berkeley, CA 94704-0163.

PJM peer review and production are managed by EditFLOW ${ }^{\circledR}$ from Mathematical Sciences Publishers.

\section{PUBLISHED BY}

\section{mathematical sciences publishers \\ nonprofit scientific publishing}

http://msp.org/

(C) 2016 Mathematical Sciences Publishers 


\section{PACIFIC JOURNAL OF MATHEMATICS}

Volume $280 \quad$ No. $1 \quad$ January 2016

Stable capillary hypersurfaces in a wedge

JAIGYOUNG CHOE and MIYUKI KoISO

The Chern-Simons invariants for the double of a compression body

DAVID L. DUNCAN

Compactness and the Palais-Smale property for critical Kirchhoff equations in

closed manifolds

EMMANUEL HeBEy

On the equivalence of the definitions of volume of representations

SUNGWOON KIM

Strongly positive representations of even GSpin groups

YEANSU KIM

An Orlik-Raymond type classification of simply connected 6-dimensional torus manifolds with vanishing odd-degree cohomology

\section{SHINTARÔ KUROKI}

Solutions with large number of peaks for the supercritical Hénon equation

Zhongyuan LiU and ShuangJie Peng

Effective divisors on the projective line having small diagonals and small heights and 141 their application to adelic dynamics

YÛSUKE OKUYAMA

Computing higher Frobenius-Schur indicators in fusion categories constructed from inclusions of finite groups

\section{Peter Schauenburg}

Chordal generators and the hydrodynamic normalization for the unit ball

SEBASTIAN SCHLEISSINGER

On a question of A. Balog

ILYA D. SHKREDOV

Uniqueness result on nonnegative solutions of a large class of differential inequalities 241 on Riemannian manifolds

YUHUA SUN

Correction to "Closed orbits of a charge in a weakly exact magnetic field"

WILL J. MERRY 